\title{
POSSÍVEIS INDICADORES DA ASSISTÊNCIA OBSTÉTRICA A PARTURIENTE EM UMA MATERNIDADE ESCOLA
}

Mariana da Silva de Figueiredoํㄹ Dayanne Kallyne Morais de Araújo Oliveira², Natália Raiane Silva Vieira², Rejane Marie Barbosa Davim ${ }^{4}$, Richardson Augusto Rosendo da Silva ${ }^{4}$

RESUMO: Trata-se de uma pesquisa quantitativa descritiva realizada em uma maternidade escola do Município de Natal, Rio Grande do Norte e teve como objetivo caracterizar a assistência obstétrica à parturiente através dos indicadores tipo de parto, presença do acompanhante durante o trabalho de parto, presença do acompanhante na sala de parto, amamentação em sala de parto, encaminhamento ao alojamento conjunto, acolhimento, privacidade e estrutura física. A amostra constou de 351 mulheres em 12 horas pós-parto e a pesquisa foi aprovada e respeitou preceitos éticos. Como resultado verificou-se que a maioria das parturientes era jovem, entre 20 e 30 anos (56\%), predominância de partos cesarianos (65\%) e ausência de acompanhantes em sala de parto (76\%). Conclui-se que é necessária atender à legislação específica, oferecendo atenção integral à saúde da mulher, privacidade, conforto, acolhimento, escuta ativa e resolutiva.

DESCRITORES: Qualidade da assistência à saúde; Parto obstétrico; Assistência ao parto; Enfermagem.

\section{POSSIBLE INDICATORS OF THE OBSTETRIC ASSISTANCE FOR THE PARTURIENT WOMAN IN A MATERNITY SCHOOL}

ABSTRACT: This quantitative descriptive research was undertaken in a maternity school in the city of Natal in the state of Rio Grande do Norte, Brazil. and aimed to characterize the obstetric assistance given to the parturient woman through indicators such as: type of birth, the presence of the companion during the labor, the presence of the companion in the delivery room, breastfeeding in the delivery room, being taken to the shared accommodation, embracement, privacy and physical structure. The sample was made up of 351 women at 12 hours post-partum, and the research was approved and respected ethical precepts. As a result, it was verified that the majority of the parturient women were young, between 20 and 30 years old $(56 \%)$, that cesarean births predominated $(65 \%)$ as did the absence of the companions in the delivery rooms $(76 \%)$. It is concluded that it is necessary to respect the specific legislation, offering the women comprehensive health care, privacy, comfort, embracement and active and resolutive listening.

DESCRIPTORS: Quality of health care; Obstetric birth; Assistance in birth; Nursing.

\section{POSIBLES INDICADORES DE LA ASISTENCIA OBSTÉTRICA A PARTURIENTA EN UNA MATERNIDAD ESCUELA}

RESUMEN: Esta es una investigación cuantitativa descriptiva realizada en una maternidad escuela del Municipio de Natal, Rio Grande do Norte, y tuvo como objetivo caracterizar la asistencia obstétrica a la parturienta a través de los indicadores tipo de parto, presencia del acompañante durante el trabajo de parto, presencia del acompañante en la sala de parto, amamantamiento en sala de parto, hecho de encaminar al alojamiento conjunto, acogimiento, privacidad y estructura física. La muestra abarcó 351 mujeres en 12 horas posparto y la investigación fue aprobada y obedeció preceptos éticos. Como resultado, se verificó que la mayoría de las parturientas era joven, entre 20 y 30 años (56\%), predominancia de partos cesáreos $(65 \%)$ y ausencia de acompañantes en sala de parto (76\%). Se concluye que es necesario atender a la legislación específica, ofreciendo atención integral a la salud de la mujer, privacidad, conforto, acogimiento, escucha activa y resolutiva. DESCRIPTORES: Cualidad de la asistencia a la salud; Parto obstétrico; Asistencia al parto; enfermería.

\footnotetext{
${ }^{1}$ Acadêmica do Curso de Graduação em Enfermagem da Universidade Federal do Rio Grande do Norte - UFRN. Bolsista PIBIC/CNPq. ${ }^{2}$ Enfermeira. Especializanda em Enfermagem em Urgência e Emergência pelas Faculdades Integradas de Patos.

${ }^{3}$ Acadêmica do Curso de Graduação em Enfermagem da UFRN. Bolsista PROEX/UFRN.

${ }^{4}$ Enfermeiro. Doutor em Ciências da Saúde. Professor do Departamento de Enfermagem e do Programa de Pós-Graduação em Enfermagem da UFRN.
}

Autor correspondente:

Richardson Augusto Rosendo da Silva

Recebido: 11/03/2013

Universidade Federal do Rio Grande do Norte

Aprovado: 17/07/2013

Rua São Clemente, 3306 - 59065-610 - Natal-RN-Brasil.

E-mail: rirosendo@yahoo.com.br

Cogitare Enferm. 2013 Out/Dez; 18(4):722-8 


\section{INTRODUÇÃO}

O processo do trabalho de parto é um fenômeno fisiológico na vida da mulher no qual há mudanças intensas, permeado por diversos sentimentos. Diante dessa realidade o Ministério da Saúde do Brasil lançou o Programa de Humanização no Pré-Natal e Nascimento (PHPN), onde entre as recomendações está à garantia de um parto seguro com o direito da parturiente ter um acompanhante no momento do parto, permanecer em alojamento conjunto com a criança, em ambiente acolhedor e que proporcione bem-estar físico e emocional ao longo do processo de parturição ${ }^{(1)}$.

O momento do parto envolve não somente a mulher e a equipe de saúde, mas sua família. As condutas para um parto humanizado devem estar focadas nas necessidades da mulher como o respeito no processo de parturição, se possível acontecer sem intervenções e reconhecer o saber nativo de sua cultura perante o contexto de vida ${ }^{(2)}$. Nesta temática a Lei 11.108/2005 assegura a parturiente o direito de ter ao seu lado um acompanhante de sua escolha no seu trabalho de parto, parto e pós-parto imediato seja no Sistema Único de Saúde (SUS) ou na rede privada ${ }^{(3)}$.

O sucesso do parto humanizado depende de adequações quanto à qualidade da assistência e humanização ao parto. Estes elementos estão fundamentados na oferta de um ambiente acolhedor que dispõe de conforto, seja silencioso, e que proporcione tranquilidade e restaure as funções corpóreas da parturiente, além de ser acolhedor para o acompanhante e equipe profissional ${ }^{(4)}$.

Nessa perspectiva o presente estudo objetivou caracterizar a assistência obstétrica à parturiente em uma maternidade escola por meio de indicadores que representam uma assistência humanizada no parto.

\section{MÉTODO}

Trata-se de um estudo descritivo quantitativo desenvolvido durante os meses de novembro de 2011 a abril de 2012 no alojamento conjunto (AC) de uma maternidadena cidade de Natal-RN complexo hospitalar da Maternidade Escola Januário Cicco (MEJC) da UFRN que participa do programa ministerial Hospital Amigo da Criança.

O AC é composto por 26 leitos, aloja em média 330 puérperas/mês, totalizando 3960 puérperas/ano. A amostra foi constituída de puérperas admitidas no AC da maternidade do estudo de forma aleatória simples do tipo proporcional com um erro amostral de $5 \%$ e confiabilidade de $95 \%$, constituindo uma amostra de
351 participantes. Foi considerado critério de inclusão estar no período de, no mínimo, 12 horas de puerpério fisiológico; justifica-se este período por considerar-se que a partir desse período as mulheres estão internadas no $\mathrm{AC}$ e em condições físicas e emocionais para responderem o questionário utilizado nesta pesquisa.

O instrumento para coleta dos dados constou de um formulário composto por duas etapas: a primeira relacionada às variáveis sócio demográficas das entrevistadas e a segunda quanto à caracterização da assistência obstétrica, utilizando-se os indicadores: tipo de parto, presença do acompanhante durante o trabalho de parto, presença do acompanhante em sala de parto, amamentação na sala de parto, encaminhamento ao $\mathrm{AC}$, satisfação com a privacidade, satisfação com o acolhimento e satisfação com a estrutura física.

Foram considerados os princípios éticos dispostos na Resolução 196/96 do Conselho Nacional de Saúde. Previamente à coleta de dados, a pesquisa foi aprovada pela instituição e pelo Comitê de Ética em Pesquisa da UFRN sob protocolo de n. 034/11 e CAAE 0043.0.051.000-11. Os dados foram coletados no AC da instituição pela equipe do projeto treinada para a realização das entrevistas.

Os resultados coletados foram organizados em banco de dados eletrônico por meio de digitação em planilha do aplicativo Microsoft Excel. Nesse processo parte dos dados foram codificados e tabulados em forma de figuras, com suas respectivas distribuições em frequências e percentuais, utilizando-se a estatística simples.

\section{RESULTADOS}

Em relação à idade, 56\%(196) das puérperas deste estudo tinham entre 20 e 30 anos, $15 \%$ eram menores de 19 anos (52) e $29 \%$ maiores de 30 anos (103). União estável foi referida por $40 \%(\mathrm{n}=140)$, seguida por solteira $37 \%(\mathrm{n}=129)$, casada $23 \%(\mathrm{n}=81)$, e divorciadas e separadas que não somaram $1 \%$ de representatividade. Quanto à renda familiar $21 \%(\mathrm{n}=73)$ obtém renda menor que um salário mínimo, $63 \%(\mathrm{n}=220)$ possui entre um e dois salários mínimos, $11 \%(\mathrm{n}=39)$ entre dois e três salários mínimos, $4 \%(\mathrm{n}=14)$ mais de três salários mínimos e $1 \%$ não souberam responder.

O grau de escolaridade das puérperas foi classificado como: não alfabetizadas $(1 \%, \mathrm{n}=3)$, estudo até a $4^{a}$ série $(13 \%, n=47)$, estudo até a $8^{a}$ série $(28 \%, n=97)$ e $18 \%(n=64)$ referiram ensino médio incompleto. A maioria concluiu o ensino médio correspondendo a $35 \%(n=122)$ da amostra e $5 \%(n=18)$ referiram ter ensino superior ou estar cursando. 
As ocupações mencionadas foram do lar $(54 \%, \mathrm{n}=191)$, estudante $(7 \%, \mathrm{n}=24)$, agricultora $(5 \%, \mathrm{n}=19)$ e outras ocupações somaram 33\% (n=117). Quanto à procedência $45 \%(n=157)$ das entrevistadas eram de Natal, $52 \%(\mathrm{n}=183)$ do interior do estado, e $4 \%(\mathrm{n}=11)$ eram procedentes de outros estados. Em relação aos dados obstétricos das entrevistadas $97 \%(\mathrm{n}=341)$ afirmaram que realizaram o pré-natal e as que não realizaram atribuíram como justificativa a falta de oportunidade; apenas $36 \%$ das multíparas realizaram o mínimo de seis consultas. Porém, quando analisado o número de gestações, $35 \%(n=124)$ eram primigestas, $28 \%(n=100)$ gesta II, e $37 \%(\mathrm{n}=127)$ multigesta. A paridade das mães equivaleu a $42 \%(\mathrm{n}=147)$ primíparas, $31 \%(\mathrm{n}=108)$ secundípara e $27 \%(\mathrm{n}=96)$ multíparas; $27 \%(\mathrm{n}=91)$ puérperas referiram ter sofrido um ou mais abortos.

No universo de partos realizados a maioria $(65 \%, n=227)$ foi do tipo cesário (Figura 1).

A caracterização da assistência obstétrica quanto aos indicadores está representada na figura 2.

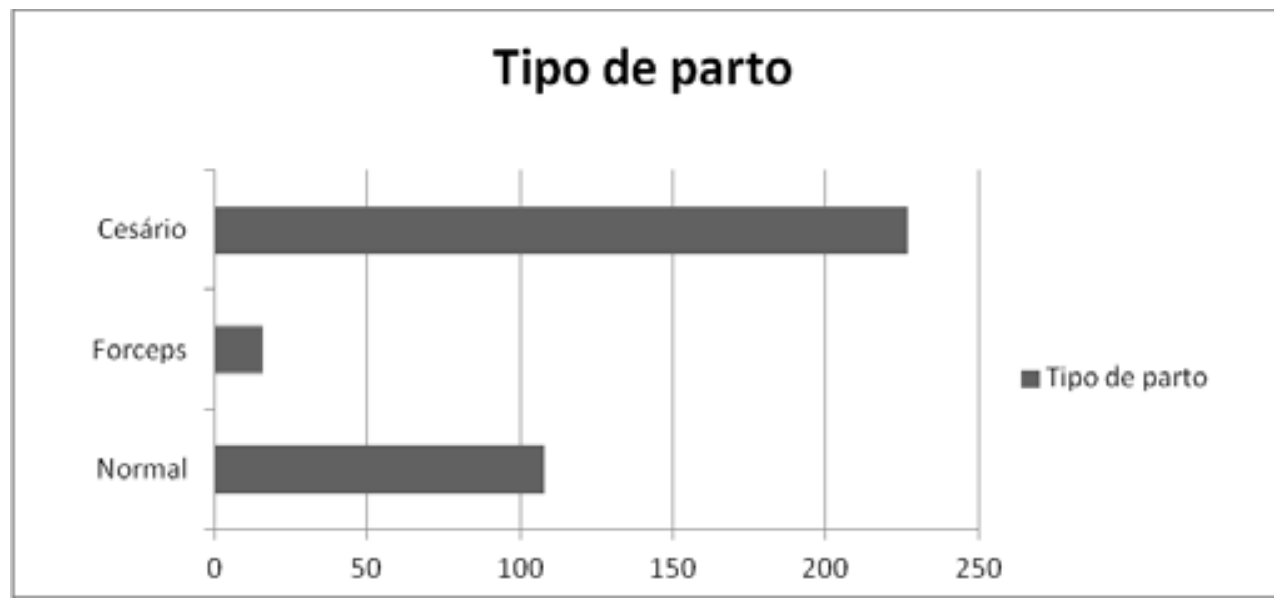

Figura 1- Distribuição de puérperas quanto ao tipo de parto realizado. Natal, 2012

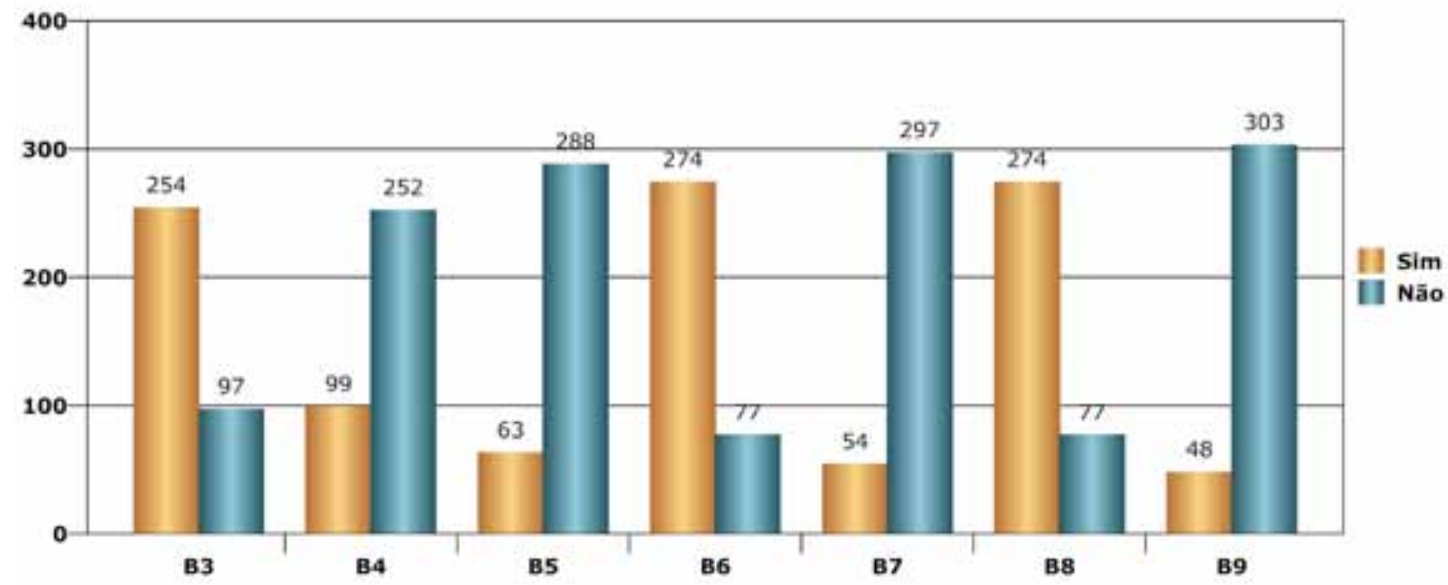

B3 - Presença do acompanhante durante o trabalho de parto; B4 - Presença do acompanhante em sala de parto; B5 - Amamentação na sala de parto; B6 - Encaminhamento ao alojamento conjunto; B7 - Satisfação com a privacidade; B8 - Satisfação com acolhimento; B9 - Satisfação com a estrutura física.

Figura 2- Indicadores da assistência obstétrica. Natal, 2012

Foram referidos como acompanhantes membros da família, entre marido, mãe e irmã. Com relação à amamentação, somente $18 \%$ referiram amamentar o bebê na sala de parto, entre as que não amamentaram, o principal motivo referido foi o fato da equipe de saúde não ter oferecido $(32 \%, n=91)$ seguido pelo fato do bebê ter necessitado de cuidados especiais $(37 \%, \mathrm{n}=108)$; o restante das participantes não soube informar o porquê não amamentou neste momento. Outro indicador analisado constatou que a maioria dos RN permaneceu em AC junto com a mãe e os que não permaneceram justificou-se devido ao bebê necessitar 
de cuidados especiais, como de oxigênio suplementar e encaminhamento à Unidade de Terapia Intensiva.

Das 351 mulheres $78 \%(n=274)$ sentiram-se bem acolhidas, apenas $15 \%(\mathrm{n}=54)$ afirmaram ter tido privacidade e somente $14 \%(\mathrm{n}=48)$ apontaram a estrutura física como adequada para o momento de parturição.

\section{DISCUSSÃO}

Ao analisar as características sociodemográficas das puérperas deste estudo observaram-se resultados comuns à população geral de usuárias de maternidades públicas no Brasil. Nesse sentido, a faixa etária com maior predominância esteve entre mulheres jovens, donas de casa que viviam em união estável com renda familiar entre um e dois salários mínimos, ensino médio completo e proviam do interior do estado. Já em um estudo realizado em Fortaleza-CE revelou que apesar das mães possuírem entre 21 e 30 anos, residiam na capital e viviam em união estável, com ensino médio incompleto $^{(5)}$.

Ao analisar os dados obstétricos observou-se no presente estudo que a maioria das entrevistadas eram multigestas e primíparas corroborando com o estudo sobre puérperas em $\mathrm{AC}^{(5)}$. No entanto um estudo realizado em uma maternidade pública de grande porte no Rio de Janeiro a proporção de mulheres nulíparas apresenta-se em elevado percentual ${ }^{(6)}$. É importante ressaltar que enquanto a taxa de natalidade no Rio Grande do Norte é de 17,9\%, no Rio de Janeiro é 11,9\%, associado a fatores sociais, culturais e econômicos. Sabe-se que quanto maior a renda e escolaridade, menor é a fecundidade, sendo uma tendência observada nas regiões Sul e Sudeste do Brasil.

As mulheres que realizaram o acompanhamento pré-natal eram, em sua maioria, multigestas, porém apenas $36 \%$ realizaram o mínimo de seis consultas. $\mathrm{O}$ acompanhamento da mulher no ciclo grávido-puerperal deve ser iniciado o mais precocemente possível. É preconizado que a gestante realize no mínimo seis consultas de pré-natal. Uma boa assistência pré-natal contribui para a diminuição dos coeficientes de mortalidade materna e infantil e promove a diminuição dos riscos das intercorrências clínico-obstétricas mais comuns durante a gestação ${ }^{(7)}$.

Em relação ao tipo de parto identificou-se grande prevalência de parto cesáreo justificado por ser uma instituição de referencia para todo o estado do Rio Grande do Norte em gestação de alto risco, além disso, existe um grande número de gestantes referenciadas provenientes do interior do estado pela falta de estrutura física e equipe especializada na assistência ao parto. Segundo o MS o índice de cesarianas vem aumentando consideravelmente por todo o país, e este tipo de parto é responsável por diversas complicações maternas e neonatais ${ }^{(7)}$. Ressalta-se que o tipo de parto, normal ou cesáreo, interfere no tempo entre o nascimento e a primeira $\operatorname{mamada}^{(8)}$. Foi evidenciado que as mães não apresentaram complicações no puerpério, porém 37\% dos RN tiveram como única complicação a dificuldade respiratória. De forma a justificar essa afirmativa um estudo de corte retrospectivo observou que a cesárea ofereceu maior risco de complicações comparado ao parto vaginal ${ }^{(9)}$.

Das usuárias entrevistadas a maioria estava acompanhada durante o seu trabalho de parto com algum familiar, no entanto algumas afirmaram que não tiveram o direito de ter um acompanhante durante o parto. Ressalta-se que na maternidade na qual foi desenvolvido o estudo é rotina a não permissão dos acompanhantes em centro cirúrgico. As pesquisas atuais mostram que na presença de um acompanhante de sua escolha, as mulheres realizam um trabalho de parto mais rápido ${ }^{(10)}$, se sentem protegidas, influenciando na menor frequência de cesarianos e partos complicados o que contribui para a mulher perceber o parto como uma experiência positiva, isto promove um melhor vínculo e ajuda no processo de aleitamento materno. Além disso, a Politica de Humanização preconiza que os acompanhantes são o elo entre o usuário, sua rede social e os demais serviços da rede de saúde com objetivo de alcançar acolhimento e segurança ao paciente ${ }^{(11)}$.

O papel do acompanhante é participar de todo o processo de parto, realizando massagens relaxantes como forma de terapia para dor, estimular a ligação afetiva promovendo a secção do cordão umbilical, e principalmente ofereça segurança à mulher. É estimulada sua participação em todos os procedimentos do trabalho de parto e parto orientando ao acompanhante com esclarecimentos de dúvidas decorrentes de condutas e procedimentos, ajudam a superar medos, ansiedades e tensões da parturiente. A equipe deve receber com respeito à parturiente e seu acompanhante, levando em conta seus gostos, opiniões e carências ${ }^{(12)}$. Em um estudo realizado em um hospital público em Fortaleza-CE o acompanhante no momento parto se mostrou de grande importância para as parturientes; sua participação foi avaliada como uma estratégia positiva e se mostrou imprescindível para garantir conforto e bem-estar às mulheres no momento da parturição ${ }^{(13)}$. 
Com relação à amamentação, poucas mulheres amamentaram na sala de parto sendo justificado por dificuldades como a cesariana, ou pelo fato de seus filhos necessitarem de cuidados especiais. Houve ainda a falta de incentivo da equipe de saúde ao aleitamento materno precoce, o que se evidencia com o desenvolver deste estudo que parece existir uma lacuna entre o que é preconizado e o que é praticado nas instituições. A Organização Mundial da Saúde (OMS) no ano de 2010 institui que toda instituição que participa da iniciativa Hospital Amigo da Criança deve ter como norma que $80 \%$ dos partos normais e pelo menos $50 \%$ das cesarianas as mulheres recebam ajuda para amamentar na primeira meia hora após o nascimento. É dada preferência que isto ocorra na sala de parto ou no centro cirúrgico, e assim favorece um maior vínculo proporcionando um contato olho no olho, pele a pele ainda em sala de parto ${ }^{(14)}$.

Um estudo realizado em uma unidade TécnicoCientífica da Fundação Oswaldo Cruz e que participa da iniciativa Hospital Amigo da Criança constatou que o tempo médio entre o nascimento e a primeira mamada é longo e principalmente quando se tratou de cesariana. Ainda no mesmo estudo, das mães que amamentaram, observando-se que o profissional de enfermagem esteve presente auxiliando as genitoras ${ }^{(4)}$. Nesse sentido, os profissionais de enfermagem possuem um papel importante nesse momento da assistência, estes são responsáveis por favorecer este momento, ou mesmo esclarecer a mãe quando isto não for possível. As orientações e o incentivo ao contato precoce favorecem ao seguimento do aleitamento materno ${ }^{(15)}$.

A equipe multiprofissional que assiste ao parto, além de ajudar no processo de aleitamento materno deve propor atividades que reduzam a ansiedade do parto, fornecer informações sobre aquele momento e sempre perguntar a opinião da parturiente. Porém algumas equipes ainda interferem no trabalho de parto, mudando o seu desencadear ${ }^{(16)}$. O cuidado deve ser proporcionado com relações menos autoritárias, resgatando a autonomia da mulher, já que se trata de um período de fragilidade, todas as condutas e orientações tomadas devem ser administradas com cautela para que tenha entendimento tanto da parturiente quanto do acompanhante ${ }^{(10-17)}$. Essa posição da equipe de enfermagem a respeito do aleitamento materno foi detectada durante a realização do presente estudo.

No que diz respeito ao direito da mãe permanecer em AC com o filho durante todo o momento de internação, o estudo evidenciou que (78\%) permaneceram junto aos seus filhos, e quando isto não aconteceu foi devido a complicações maternas ou neonatais, pois é política da instituição que a mãe permaneça junto ao recém nascido no mesmo ambiente. $\mathrm{O} A C$ além de proporcionar o incentivo ao aleitamento materno possibilita o aumento do vínculo entre a mãe, o $\mathrm{RN}$ e a família, proporcionando uma assistência integral e humanizada. Outro aspecto são os benefícios para as mães, pois tais ações favorecem a aceitação da maternidade, proporcionando à puérpera um sentimento de bem-estar e praticidade, e favorece a troca de experiência entre as mães ${ }^{(18)}$.

Para os indicadores satisfação com o acolhimento, estrutura física e privacidade as mães afirmaram sentir-se bem acolhidas, no entanto a maioria não sentiu satisfação com a estrutura física e referiu falta de privacidade, aspectos que interferem diretamente na qualidade da assistência oferecida. Nesse sentido, é importante para a puérpera ter um ambiente acolhedor, que apresente leitos suficientes, banheiros individuais, boa iluminação e privacidade. Para pensar a estrutura adequada no AC faz-se necessário relacionar o espaço físico às necessidades das mães no período do puerpério, levando em consideração a importância da adaptação do ambiente, a fim de aperfeiçoar o funcionamento do serviço. Essa perspectiva pode favorecer a integralidade do cuidado à saúde e promover o bem estar tanto das usuárias quanto da equipe de saúde.

Humanizar com qualidade a atenção de saúde é responsabilidade, principalmente, dos profissionais de saúde e assim favorecer condições que resultem na identificação de problemas, satisfação das mães, capacidade de enfretamento e reconhecimento dos seus direitos ${ }^{(2)}$.

\section{CONCLUSÃO}

A caracterização da assistência obstétrica a parturiente foi demonstrada pela prevalência de parto cesariano, acompanhantes durante o trabalho de parto, ausência do acompanhante e da prática da amamentação em sala de parto, insatisfação com a privacidade e estrutura física, no entanto as puérperas sentiram-se bem acolhidas.

É necessária uma atenção integral a mulher que permita a presença do acompanhante durante o trabalho de parto e parto, que incentive o aleitamento materno ainda em sala de parto. Essas ações visam aumentar o vinculo entre a mãe, a família e a criança que acaba de nascer e principalmente na promoção de educação em 
saúde que pode ser realizada no alojamento conjunto através das orientações. É necessário não focar só nos dados clínicos, mas também o emocional e psicológico, tornando o atendimento mais humanizado à medida que contempla sua condição como usuária autônoma e independente a participar de seu próprio parto e pós-parto. O envolvimento desta é a garantia para que a mesma possa vivenciar o processo mais importante que está acontecendo em sua vida e de sua família.

Por fim espera-se que esses achados possam contribuir para se repensar o cuidado prestado às puérperas em suas particularidades e na diversidade de situações em que se encontra e que os resultados possam estimular reflexões para que o cuidado seja planejado e construído, sempre com a finalidade de atender a parturiente de maneira integra proporcionando a estas protagonizar esse momento de vida.

\section{REFERÊNCIAS}

1. Ministério da Saúde (BR). Secretaria de Políticas de Saúde. Assistência pré-natal: manual técnico. [Internet] Brasília, 2000. [acesso em 26 out 2012]. Disponível: http://bvsms.saude.gov.br/bvs/publicacoes/cd04_11.pdf.

2. Moreira K, Araújo M, Fernandes A, Braga V, Marques J, Queiroz M. O significado do cuidado ao parto na voz de quem cuida: uma perspectiva à luz da humanização. Cogitare enferm. [Internet] 2009;14(4) [acesso em 26 out 2012]. Disponível: http://ojs.c3sl.ufpr.br/ojs2/index. php/cogitare/article/download/16389/10869

3. Brasil. Lei n.11.108, de 7 de Abril de 2005. Altera a Lei n. 8.080, de 19 de setembro de 1990, para garantir às parturientes o direito à presença de acompanhante durante o trabalho de parto, parto e pós-parto imediato, no âmbito do Sistema Único de Saúde - SUS. Brasília [Internet] 2005 [acesso em 26 out 2012]. Disponível: http://www.planalto.gov.br/ccivil_03/_Ato20042006/2005/Lei/L11108.htm.

4. Silva RC, Soares MC, Muniz RM, Andrade FP, Torres AAP, Gomes V. La concepción de los profesionales de salud sobre el parto humanizado en la adolescencia. Enferm. glob. [Internet] 2011;10(4) [acesso em 18 mai 2012]. Disponível: http://dx.doi.org/10.4321/S169561412011000400025

5. Dodt RCM, Oriá MOB, Pinheiro AKB, Almeida PC, Ximenes LB. Perfil Epidemiológico das Puérperas AssistidasemumAlojamentoConjunto. Rev.enferm.UERJ. [Internet] 2010;18(3) [acesso em 17 out 2012]. Disponível: http://www.facenf.uerj.br/v18n3/v18n3a02.pdf
6. Pereira ALF, Nagipe SFSA, Lima GPV, Nascimento SD, Gouveia MSF. Cuidados e resultados da assistência na sala de relaxamento de uma maternidade pública, Rio de Janeiro, Brasil. Texto Contexto Enferm. [Internet] 2012;21(3) [acesso em 17 mai 2012] Disponível: http:// dx.doi.org/10.1590/S0104-07072012000300011

7. Ministério da Saúde (BR). Secretaria de atenção á saúde. Departamento de atenção básica. Atenção de pré-natal de baixo risco. Caderno de atenção básica [Internet] 2012;32 [acesso em 16 mai 2013]. Disponível: https:// mail-attachment.googleusercontent.com/attachment/? $\mathrm{ui}=2 \& \mathrm{ik}=533395287 \mathrm{f} \&$ view $=$ att $\& \mathrm{th}=13 \mathrm{eaf} 2 \mathrm{~d} 3 \mathrm{c} 2 \mathrm{ea} 8 \mathrm{~b}$ 79\&attid $=0.1 \&$ disp $=$ inline $\&$ safe $=1 \& z$ w \& saduie $=A G$ 9B_P998C17qQbzaAwKoZElzjsQ\&sadet $=136885979$ 1478\&sads=k6VSQIfbDlIBaLt5v7XwAgny7Q4

8. Silva SC, Silva LR, Mathias LFB. O tempo médio entre o nascimento e a primeira mamada: o ideal e o real. Rev. Eletr. Enf.[Internet]2008;10(3) [acessoem 18 mai2013].Disponível: http://www.fen.ufg.br/revista/v10/n3/v10n3a11.htm

9. Machado JLC, Sevrin CE, Oliveira ECHB, Carvalho HB, Zamboni JW, Araújo JC de, et al. Associação entre via de parto e complicações maternas em hospital público da Grande São Paulo, Brasil. Cad. Saúde Pública [Internet] 2009;25(1) [acesso em 18 mai 2013]. Disponível: http://dx.doi.org/10.1590/S0102311X2009000100013

10. Brüggemann O, Parpinelli M, Osis M, Cecatti J, Neto A. Apoio à parturiente por acompanhante de sua escolha em maternidade brasileira: ensaio clínico controlado randomizado. Rev. Tempus Actas Saúde Colet. [Internet] 2010;(4). [acesso em 17 mai 2013]. Disponível: http:// tempusactas.unb.br/index.php/tempus/article/view/843/806

11. Ministério da Saúde (BR). Secretaria de Atenção à Saúde. Núcleo técnico da política nacional de humanização. HumanizaSUS : visita aberta e direito a acompanhante/ Ministério da Saúde, Secretaria de Atençãoà Saúde, Núcleo Técnico da Política Nacional de Humanização. $-2^{\mathrm{a}}$ ed. $4^{\mathrm{a}}$ reimp. Brasília [Internet] 2010 [acesso em 26 out 2012]. Disponível:http://bvsms.saude.gov.br/bvs/publicacoes/ humanizasus_visita_aberta_direito_acompanhante.pdf

12. Associação Brasileira de Obstetrizes e Enfermeiros Obstetras (ABENFO). Unidade de saúde parceira do pai. [internet] 2009;(1). [acesso em 16 mai 2013]. Disponível: http://abenfo.redesindical.com.br/arqs/manuais/047.pdf

13. Oliveira ASS, Rodrigues DP, Guedes MVC, Felipe GF, Galiza FT, Monteiro LC. O acompanhante no momento do trabalho de parto e parto: percepção de puérperas. Cogitare enferm. [Intenet] 2011;16(2) [acesso em 26 
out 2012]. Disponível: http://ojs.c3sl.ufpr.br/ojs-2.2.4/ index.php/cogitare/article/viewFile/20201/14211

14. Organização Mundial de Saúde. Iniciativa hospital amigo da criança: revista, atualizada e ampliada para o cuidar integrado, módulo 4, autoavaliação e monitoramento do hospital. Brasília: OMS [Internet] 2010 [acesso em 15 mai 2013]. Disponível: http://bvsms. saude.gov.br/bvs/publicacoes/iniciativa_hospital amigo_crianca_modulo4.pdf

15. Junges CF, Ressel LB, Budó MLD, Padoin SMM, Hoffmann IC, Sehnem GD. Percepções de puérperas quanto aos fatores que influenciam o aleitamento materno. Rev. Gaúcha Enferm. [Internet] 2010;31(2) [acesso em 17 mai 2013]. Disponível: http://dx.doi. org/10.1590/S1983-14472010000200020

16. Longo CSM, Andraus LMS, Barbosa MA. Participação do acompanhante na humanização do parto e sua relação com a equipe de saúde. Rev. Eletr. Enf. [Internet] 2010;12(2) [acesso em 16 mai 2013]. Disponível: http://www.revistas.ufg.br/index.php/fen/article/ view/5266/6945

17. Bezerra CP, Machado MMT, Sousa LB. Humanização da assistência ao parto: concepções e percepções da equipe de enfermagem. In: $7^{\circ}$ Congresso Brasileiro de Enfermagem Obstetra e Neonatal-COBEON; 2011. p. 5311-24; Belo Horizonte, Brasil. Belo Horizonte: COBEON; 2011 [acesso em 17 mai 2013]. Disponível: http://www.redesindical.com.br/abenfo/viicobeon_ icieon/files/0604.pdf

18. Pasqual KK, Braccialli LA, Volponi M. Alojamento conjunto: espaço concreto de possibilidades e o papel da equipe multiprofissional. Cogitare enferm. [Internet] 2010;15(2) [acesso em 12 mai 2012]. Disponível: http://ojs.c3sl.ufpr.br/ojs2/index.php/cogitare/article/ view/17872/11662

Cogitare Enferm. 2013 Out/Dez; 18(4):722-8 